\title{
On Smooth Multivariate Spline Functions
}

\author{
By Charles K. Chui* and Ren-Hong Wang**
}

\begin{abstract}
In this paper the dimensions of bivariate spline spaces with simple cross-cut grid partitions are determined and expressions of their basis functions are given. Consequently, the closures of these spaces over all partitions of the same type can be determined. A somewhat more detailed study on bivariate splines with rectangular grid partitions is included. The results in this paper can be applied to problems on interpolation and approximation by bivariate spline functions
\end{abstract}

1. Introduction. During the past three and a half decades a vast amount of work has been done on univariate spline functions and their computational as well as approximation properties. Of central importance are, perhaps, $B$-splines (or basic splines) first studied in some detail by I. J. Schoenberg [22]: they not only serve as bases of spline spaces, but, with the ingenious simple normalization introduced by $\mathrm{C}$. de Boor [4], also provide very efficient spline interpolants and approximants both for computational and theoretical considerations. One of the most important properties of the normalized $B$-splines of de Boor is that they form a locally supported partition of unity. This fact has been used, for instance, in the investigation of the order of monotone approximation by splines [2], [8], [9], [15]. A fairly complete survey on the subject of univariate $B$-splines was given by de Boor [5].

In [5], de Boor also introduced the notion of multivariate $B$-splines which are locally supported nonnegative $C^{k-1}$ piecewise polynomials of total degree $k$. It was C. A. Micchelli [19], [20] who developed the very elegant theory of these multivariate $B$-splines. In addition, W. Dahmen [12] also provided their truncated power representations. See also [14] for more information. It should be noted, however, that these multivariate $B$-splines are determined by a given set of "knots" instead of grid lines. In fact, the "knots" determine certain simplices which in turn give the grid lines that separate the polynomial pieces. Hence, there are generally a fairly large number of grid lines. In this paper, we are interested in the study of multivariate spline functions that are directly determined by a given grid configuration. More precisely, we will consider multivariate spline spaces $S_{k}^{\mu}(\Delta)$ consisting of all $C^{\mu}$ functions which are polynomials of total degree $k$ in each of the cells of the grid partition $\Delta$. It is important to know the dimensions of these spaces and give explicit

Received July 29, 1981; revised March 1, 1982 and August 23, 1982.

1980 Mathematics Subject Classification. Primary 41A15, 41A63.

$K e y$ words and phrases. Multivariate spline functions, total degree, $B$-splines, conformality condition, basis, cross-cuts.

*The work of this author was supported by the U.S. Army Research Office under Grant No. DAAG 29-78-0097 and Contract No. DAAG 29-81-K-0133.

${ }^{* *}$ This author was a visiting scholar at Texas A \& M University during 1981-1982. 
expressions of their basis functions. Several conjectures on the dimension of $S_{k}^{\mu}(\Delta)$ where $\Delta$ is a certain triangulation have been made by G. Strang [26], [27]. Also, L. L. Schumaker has studied the dimension problem in [25]. In this paper, the basis problem of $S_{k}^{\mu}(\Delta), 0 \leqslant \mu<k$, will be studied with emphasis on locally supported basis functions. When the grid lines are "simple" cross-cuts, we give explicit expressions of their basis functions. It will be seen that if $\Delta$ is fairly "simple" and $\mu$ is "large", no locally supported basis functions exist. More surprisingly, if both fairly smooth splines are desirable and fairly "simple" grid partitions $\Delta$ are to be used, then the closure (in the topology of the given normed linear function space $X$ ) of the union of $S_{k}^{\mu}(\Delta)$ over all "simple" grid partitions of the same nature is a fairly "thin" subset of $X$. These "thin" subsets will be determined explicitly for partitions with cross-cut grid lines. Therefore, as a rule of thumb, good approximants are obtained only when $\mu$ is small or else $\Delta$ is allowed to be more "complicated". When no smoothness condition is required, direct and inverse estimates were given by $\mathrm{W}$. Dahmen, R. DeVore, and K. Scherer [13], and Dupont and Scott [16]. Also, it is well known that much work has been done on tensor product spline approximation (see for example [6], [21], [23], [24], [32].) Our techniques in the preliminary results are extensions of those used by the second author [29]-[31] in the study of existence and representation of multivariate splines under very general grid partitions. The problems we are interested in are of a different nature than those on spline-blended surface interpolation (see, for example [1], [3], [11], [17], [18]). In order to give as clear a presentation as possible, only spline functions of two variables will be discussed. It will be clear that our techniques and results can be easily generalized to an arbitrary multi-dimensional setting.

2. Preliminary Results. Let $D$ be a domain in $\mathbf{R}^{2}$ and $\Delta$ a grid of curves that divide $D$ into a finite number of cells. Each boundary curve segment that separates two adjacent cells will be called a grid-segment (or edge), and the two endpoints of a grid-segment will be called grid-points. Hence, grid-points are points of intersection of all the curves that determine the grid $\Delta$. Let $\mathbf{P}_{k}$ denote the collection of all polynomials with real coefficients and total degree $k$; that is, each $p \in \mathbf{P}_{k}$ has the representation

$$
p(x, y)=\sum_{0 \leqslant i+j \leqslant k} c_{i j} x^{i} y^{j}
$$

where $c_{i j}$ are real numbers. A function $s$ in $C^{\mu}(D), 0 \leqslant \mu \leqslant k-1$, will be called a multivariate (or, more precisely, bivariate) spline function of (total) degree $k$ (or order $k+1$ ) and with smoothness joining condition $C^{\mu}$ determined by a grid partition $\Delta$, if the restriction of $s$ to each cell of this partition is in $\mathbf{P}_{k}$. The collection of all these bivariate spline functions will be denoted by $S_{k}^{\mu}=S_{k}^{\mu}(\Delta)=S_{k}^{\mu}(\Delta ; D)$. We also use the notation $S_{k}=S_{k}^{k-1}$. In the following argument, we will assume that every grid-segment (or edge) $\Gamma_{i j}$ that separates two adjacent cells $D_{i}$ and $D_{j}$, say, is a segment of an algebraic curve: that is, $\Gamma_{i j}$ is defined by $l_{i j}(x, y)=0$, where $l_{i j}$ is a polynomial in $x$ and $y$. Throughout, all such polynomials $l_{i j}$ will be assumed to be irreducible over the real field. Note that $\Gamma_{i j}=\Gamma_{j i}$ and $l_{i j}=l_{j i}$. The following old result of Bezout (cf. [28]) will be used in this paper. 
LEMMA 2.1. If the number of common zeros of two real-valued polynomials in two real variables is greater than the product of their total degrees, then these two polynomials must have a nontrivial common factor.

As an application, we observe that if

$$
\Gamma: l(x, y)=0
$$

is a grid-segment (where $l$ is an irreducible polynomial), then $\operatorname{Grad} l(x, y)$ has at most a finite number of zeros on $\Gamma$. Indeed, if the contrary holds, then $l$ has nontrivial common factors with both $\partial l / \partial x$ and $\partial l / \partial y$. Since $l$ is irreducible, $l$ must divide both $\partial l / \partial x$ and $\partial l / \partial y$, so that both $\partial l / \partial x$ and $\partial l / \partial y$ must be identically zero, or $l$ is a constant, which is not possible. As another application of Lemma 2.1, we have the following result.

LeMma 2.2. Let $D_{i}$ and $D_{j}$ be two adjacent cells of a partition $\Delta$ with common grid-segment $\Gamma_{i j}$ defined by $l_{i j}(x, y)=0$. If $s \in S_{k}^{\mu}, 0 \leqslant \mu<k$, and the restrictions of $s$ on $D_{i}$ and $D_{j}$ are polynomials $p_{i}$ and $p_{j}$ in $\mathbf{P}_{k}$, respectively, then

$$
p_{i}-p_{j}=\left[l_{i j}\right]^{\mu+1} Q_{i j},
$$

where $Q_{i j}$ is a polynomial.

We will call $\left[l_{i j}\right]^{\mu+1}$ the smoothing factor and $Q_{i j}$ the smoothing cofactor of the bivariate spline function $s$ across the grid-segment $\Gamma_{i j}$ from $D_{i}$ and $D_{j}$. Note that since $\Gamma_{i j}=\Gamma_{j i}$ or $l_{i j}=l_{j i}$, we have

$$
Q_{i j}=-Q_{j i}
$$

To prove this lemma, we first observe that

$$
\frac{\partial^{r}}{\partial x^{l} \partial y^{r-1}}\left[p_{i}(x, y)-p_{j}(x, y)\right]=0
$$

for $(x, y) \in \Gamma_{i j}, l=0, \ldots, r$ and $r=0, \ldots, \mu$. Applying (2.2) for $r=0$, we see that $\left(p_{i}-p_{j}\right)$ and $l_{i j}$ are both zero on $\Gamma_{i j}$ and must have a common factor by Lemma 2.1. Since $l_{i j}$ is irreducible, we have

$$
p_{i}-p_{j}=l_{i j} T_{1}
$$

for some polynomial $T_{1}$. Next,

$$
\frac{\partial}{\partial x}\left(p_{i}-p_{j}\right)=T_{1} \frac{\partial}{\partial x} l_{i j}+l_{i j} \frac{\partial}{\partial x} T_{1}
$$

and

$$
\frac{\partial}{\partial y}\left(p_{i}-p_{j}\right)=T_{1} \frac{\partial}{\partial y} l_{i j}+l_{i j} \frac{\partial}{\partial y} T_{1}
$$

Since Grad $l_{i j}$ has at most a finite number of zeros on $\Gamma_{i j}$, as we observed earlier, we must have $T_{1}(x, y)=0$ for all $(x, y) \in \Gamma_{i j}$ by using (2.2) for $r=1$. Hence

$$
T_{1}=l_{i j} T_{2}
$$

for some polynomial $T_{2}$, or

$$
p_{i}-p_{j}=\left[l_{i j}\right]^{2} T_{2}
$$


Moreover, for each $r, 1 \leqslant r \leqslant \mu$, since $l_{i j}=0$ on $\Gamma_{i j}$, we have, for all $(x, y) \in \Gamma_{i j}$,

$$
\frac{\partial^{r}}{\partial x^{r}}\left[l_{i j}(x, y)\right]^{r}=r !\left[\frac{\partial}{\partial x} l_{i j}(x, y)\right]^{r}
$$

and

$$
\frac{\partial^{r}}{\partial y^{r}}\left[l_{i j}(x, y)\right]^{r}=r !\left[\frac{\partial}{\partial y} l_{i j}(x, y)\right]^{r} .
$$

Hence, by repeating the above procedure $\mu-1$ times and applying (2.2) for $l=0$ and $r, r=2, \ldots, \mu$, we obtain (2.1) with $Q_{i j}=T_{\mu+1}$. This completes the proof of the lemma.

An important consequence of Lemma 2.2 is that if $A$ is any grid-point which is the endpoint of grid-segments $\Gamma_{i j}^{(r)}, r=1, \ldots, N$, ordered in the counter-clockwise direction around $A$, then we have

$$
\sum_{r=1}^{N}\left[l_{i j, r}(x, y)\right]^{\mu+1} Q_{i j, r}(x, y)=0
$$

for all $(x, y)$, where $\Gamma_{i j}^{(r)}$ is determined by $l_{i j, r}(x, y)=0$ and $Q_{i j, r}$ is the smoothing cofactor of the bivariate spline function $s$ across $\Gamma_{i j}^{(r)}$, again travelling in the counter-clockwise direction. For simplicity, the above identity will be denoted by

$$
\sum_{A}\left[l_{i j}(x, y)\right]^{\mu+1} Q_{i j}(x, y) \equiv 0 .
$$

We will call (2.4) the conformality condition at the grid-point $A$. This idea is a generalization of a certain constraint observed by G. Strang in his study of the dimension of $C^{1}$ piecewise polynomials on triangular meshes [26], [27].

Choose any cell $D_{*}=D_{*}(\Delta)$ of a grid partition $\Delta$ of the domain $D$, and consider $D_{*}$ as the source of a flow into all the other cells of this partition. $D_{*}$ will be called a source cell, and the flow from $D_{*}$ into the other cells is via a system of one or more main streams with branches, subbranches, subsubbranches, etc., in such a way that the flow does not pass through any grid-point and reaches every single cell of the partition in one and only one way. This requires, of course, that the streams in this flow (that is the main streams, branches, subbranches, etc.) never cross one another. Of course, the streams cross the grid-segments without touching their endpoints, the grid-points. Let $s$ be a bivariate spline in $S_{k}^{\mu}$ and $(x, y)$ any point in $D$ which is not on the grid $\Delta$. Then $(x, y)$ is in some cell $D_{(x, y)}$. If $D_{(x, y)}$ happens to be the source cell $D_{*}$, then we have

$$
s(x, y)=p_{k}^{*}(x, y),
$$

where $p_{k}^{*} \in \mathbf{P}_{k}$. If $(x, y)$ is not in $D_{*}$, then there is only one way that the flow from $D_{*}$ can reach $D_{(x, y)}$ via the system of streams described above. Let $\vec{C}=\vec{C}(x, y)$ be this stream. Also, let $\Gamma_{i j}: l_{i j}(x, y)=0$ be the grid-segments of the partition $\Delta$. We introduce the following notation:

$$
\left[l_{i j}(x, y)\right]_{*}= \begin{cases}l_{i j}(x, y) & \text { if } \vec{C}(x, y) \text { crosses } \Gamma_{i j} \\ 0 & \text { otherwise }\end{cases}
$$


Let $Q_{i j}$ be the smoothing cofactor of the bivariate spline $s$ across $\Gamma_{i j}$ from a cell $D_{i}$ to its neighboring cell $D_{j}$ (and recall that $Q_{j i}=-Q_{i j}$ although $l_{j i}=l_{i j}$ ). One direction of the next result follows immediately from Lemma 2.2.

THEOREM 2.1. Let $s$ be a bivariate spline function in $S_{k}^{\mu}, 0 \leqslant \mu<k$, whose restriction on a source cell $D_{*}$ is $p_{k}^{*} \in \mathbf{P}_{k}$. Then

$$
s(x, y)=p_{k}^{*}(x, y)+\sum\left[l_{i j}(x, y)\right]_{*}^{\mu+1} Q_{i j}(x, y),
$$

$(x, y) \in D$, where the summation is taken along the flow described above. Conversely, if the functions $Q_{i j}$ in (2.6) satisfy the conformality condition (2.4) at every grid-point, then $s$ is in $S_{k}^{\mu}$.

If $(x, y) \in D \backslash \Delta$, the above representation formula clearly holds. For $(x, y) \in \Delta$, formula (2.6) is still valid due to the continuity of $s$ on $D$. If $\Delta$ consists of only one grid-point, the converse clearly follows, and, in general, it can be established by applying mathematical induction. It should be emphasized that the formula (2.6) depends on the choice of the source cell $D_{*}$ and its flow system. However, because of the conformality conditions at all grid-points that a bivariate spline $s$ must satisfy, we could have picked any flow system to represent $s$.

3. Rectangular Grid Partition. This and the next sections will be devoted to the special case when the grid partition $\Delta$ of a rectangular domain $D$ in $\mathbf{R}^{2}$ consists of horizontal and vertical lines only. It will be clear that the following argument does not depend too much on the shape of $D$. A more general setting will be discussed in Section 4. For convenience we let $D$ be the open unit square $\{(x, y): 0<x, y<1\}$ and consider

$$
0=x_{-k}=\cdots=x_{0}<x_{1}<\cdots<x_{m}<x_{m+1}=\cdots=x_{m+k+1}=1
$$

and

$$
0=y_{-k}=\cdots=y_{0}<y_{1}<\cdots<y_{n}<y_{n+1}=\cdots=y_{n+k+1}=1 .
$$

Our grid partition $\Delta=\Delta_{m n}$ will consist of the algebraic curves: $x-x_{i}=0$ and $y-y_{j}=0, i=1, \ldots, m$ and $j=1, \ldots, n$. The cell with grid-points or vertices $\left(x_{i}, y_{j}\right)$, $\left(x_{i+1}, y_{j}\right),\left(x_{i+1}, y_{j+1}\right),\left(x_{i}, y_{j+1}\right), 0 \leqslant i \leqslant m$ and $0 \leqslant j \leqslant n$, will be denoted by $G_{i j}=\left\langle x_{i}, x_{i+1} ; y_{j}, y_{j+1}\right\rangle$.

Let $A=\left(x_{i}, y_{j}\right), 1 \leqslant i \leqslant m$ and $1 \leqslant j \leqslant n$ be any grid-point. It is the common grid-point of four cells $G_{i-1, j-1}, G_{i, j-1}, G_{i, j}, G_{i-1, j}$ which we denote by $D_{1}, D_{2}, D_{3}$, $D_{4}$, respectively. Hence, the grid-segments $\Gamma_{12}$ and $\Gamma_{34}$ are both determined by $l_{12}(x, y)=l_{34}(x, y)=x-x_{i}$ and those of $\Gamma_{23}$ and $\Gamma_{41}$ by $l_{23}(x, y)=l_{41}(x, y)=y$ $-y_{j}$. As usual, denote the corresponding smoothing cofactors of a bivariate spline $s \in S_{k}^{\mu}\left(\Delta_{m n}\right)$ by $Q_{12}(x, y), Q_{23}(x, y), Q_{34}(x, y)$, and $Q_{41}(x, y)$.

Let us first consider the case $\mu=k-1$ :

LEMMA 3.1. Let $s \in S_{k}\left(\Delta_{m n}\right)$ and $Q_{12}, Q_{23}, Q_{34}, Q_{41}$ be its smoothing cofactors as described above. Then $Q_{12}, Q_{23}, Q_{34}$, and $Q_{41}$ are constants satisfying $Q_{12}=-Q_{34}$ and $Q_{23}=-Q_{41}$.

This result follows from Lemma 2.2 and the conformality condition at the grid-point $A$. Indeed, by Lemma 2.2 the smoothing cofactors are constants, and by 
the conformality condition they satisfy

$$
\left(Q_{12}+Q_{34}\right)\left(x-x_{i}\right)^{k}+\left(Q_{23}+Q_{41}\right)\left(y-y_{j}\right)^{k} \equiv 0
$$

so that $Q_{12}=-Q_{34}$ and $Q_{23}=-Q_{41}$. By applying this lemma repeatedly, we also have the following

LEMMA 3.2. Let $c_{1}$ and $c_{2}$ be two smoothing cofactors of a bivariate spline in $S_{k}\left(\Delta_{m, n}\right)$ defined by travelling either from two cells on the left of $x-x_{i}=0$ to their corresponding neighboring cells on the right of $x-x_{i}=0$, or from two cells below $y-y_{j}=0$ to their corresponding neighboring cells above $y-y_{j}=0$, then $c_{1}=c_{2}$.

An important implication of this lemma is that the representation formula (2.6) in this setting is independent of the choice of the flow system in the sense that the smoothing cofactors for a different flow system do not change. Indeed, if a cell $D_{0}$ is on the same side of $x-x_{i}=0$ or $y-y_{j}=0$ as the source cell $D_{*}$, then any stream that flows from $D_{*}$ to $D_{0}$ crosses $x-x_{i}=0$ or $y-y_{j}=0$ an even number of times, so that all the smoothing cofactors across $x-x_{i}=0$ or $y-y_{j}=0$ cancel, and if, on the other hand, $D_{0}$ lies on the opposite side of $x-x_{i}=0$ or $y-y_{j}=0$ as $D_{*}$, then any stream flowing from $D_{*}$ to $D_{0}$ crosses $x-x_{i}=0$ or $y-y_{j}=0$ an odd number of times, so that all except one smoothing cofactors across $x-x_{i}=0$ or $y-y_{j}=0$ cancel. For convenience, let us select $G_{(0)}=\left\langle x_{0}, x_{1} ; y_{0}, y_{1}\right\rangle$ as the source cell. The above argument yields the following result.

Proposition 3.1. Any bivariate spline function $s$ in $S_{k}\left(\Delta_{m n}\right)$ has the following unique representation:

$$
s(x, y)=p_{k}^{*}(x, y)+\sum_{i=1}^{m} a_{i}\left(x-x_{i}\right)_{i}^{k}+\sum_{j}^{n} b_{j}\left(y-y_{j}\right)_{+}^{k}
$$

when $p_{k}^{*} \in \mathbf{P}_{k}$ and $a_{1}, \ldots, a_{m}, b_{1}, \ldots, b_{n}$ are real constants.

Here and throughout this paper, we use the standard notation $a_{+}=\max (a, 0)$ and $a_{+}^{k}=\left(a_{+}\right)^{k}$. For computational and other purposes, it is more convenient to use the normalized $B$-splines (cf. [4]):

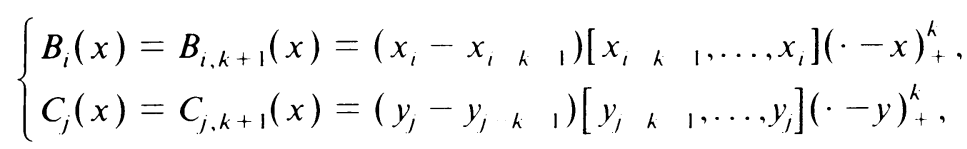

$i=1, \ldots, m+k+1$ and $j=1, \ldots, n+k+1$. Since

$$
C_{n+k+1}(y)=\sum_{i=1}^{m+k+1} B_{i}(x)-\sum_{j=1}^{n+k} C_{j}(y)
$$

for all $(x, y) \in D$, we have the following

Proposition 3.2. The bivariate spline space $S_{k}\left(\Delta_{m n}\right)$ has a basis given by $\left\{x^{u+1} y^{v+1}, B_{i}(x), C_{j}(y)\right\}$, where $u, v \geqslant 0,0 \leqslant u+v \leqslant k-2$, and $i=1, \ldots, m+k$ $+1 ; j=1, \ldots, n+k$. The dimension of $S_{k}\left(\Delta_{m n}\right)$ is $\frac{1}{2}(k+1)(k+2)+m+n$.

From the above results, it follows immediately that not too many functions on $D$ can be accurately approximated by bivariate splines in $S_{k}\left(\Delta_{m n}\right)$. For the sake of 
convenience in stating this and other results later, we will let $X$ be the Banach space $L_{p}(D)$ if $1 \leqslant p<\infty$ or $C(\bar{D})$ if $p=\infty$. We then have the following

Corollary 3.1. The closure in $X$ of the union of $S_{k}(\Delta)$ over all rectangular grid partitions $\Delta$ of $D$ is the subspace of all functions which have the form $p_{k}(x, y)+f(x)$ $+g(y)$ where $p_{k} \in P_{k}$.

With a little more care, the results on $S_{k}$ can be generalized to $S_{k}^{\mu}, 0 \leqslant \mu<k-1$. To do this, we return to the notation we introduced earlier in this section concerning $G_{i j}, A=\left(x_{i}, y_{j}\right), D_{1}, \ldots, D_{4}, \Gamma_{12}, \ldots, \Gamma_{41}$, and the smoothing cofactors $Q_{12}(x, y), \ldots, Q_{41}(x, y)$. We have the following result.

Lemma 3.3. Let $s \in S_{k}^{\mu}, 0 \leqslant \mu \leqslant k-1$, and $Q_{12}, Q_{23}, Q_{34}, Q_{41}$ be its smoothing cofactors in the counter-clockwise direction across $\Gamma_{12}, \Gamma_{23}, \Gamma_{34}, \Gamma_{41}$, respectively. Then

$$
Q_{12}(x, y)+Q_{34}(x, y)=\left(y-y_{j}\right)^{\mu+1} d(x, y)
$$

and

$$
Q_{23}(x, y)+Q_{41}(x, y)=-\left(x-x_{i}\right)^{\mu+1} d(x, y)
$$

for all $(x, y)$, where $d \in \mathbf{P}_{k-2 \mu-2}$. In particular, if $\mu>(k-2) / 2$, then $Q_{12}(x, y)+$ $Q_{34}(x, y)=Q_{23}(x, y)+Q_{41}(x, y)=0$ for all $(x, y)$.

To prove this lemma, we return to the conformality condition (2.4) and obtain

$$
\begin{aligned}
\left(Q_{12}(x, y)\right. & \left.+Q_{34}(x, y)\right)\left(x-x_{i}\right)^{\mu+1} \\
& +\left(Q_{23}(x, y)+Q_{41}(x, y)\right)\left(y-y_{j}\right)^{\mu+1} \equiv 0 .
\end{aligned}
$$

Since $\left(x-x_{i}\right)^{\mu+1}$ and $\left(y-y_{j}\right)^{\mu+1}$ are relatively prime, we have $Q_{12}+Q_{34}=$ $\left(y-y_{j}\right)^{\mu+1} T_{1}$ and $Q_{23}+Q_{41}=\left(x-x_{i}\right)^{\mu+1} T_{2}$ for some $T_{1}, T_{2} \in \mathbf{P}_{k-2 \mu-2}$. Putting these back into (3.3) gives

$$
\left(T_{1}(x, y)+T_{2}(x, y)\right)\left(x-x_{i}\right)^{\mu+1}\left(y-y_{j}\right)^{\mu+1} \equiv 0 .
$$

Hence, $T_{1}=-T_{2}=d \in \mathbf{P}_{k-2 \mu-2}$. If $\mu>(k-2) / 2$, it is clear that $T_{1}$ and $T_{2}$ are identically zero.

Next we will derive a representation formula for bivariate spline functions in $S_{k}^{\mu}$, $0 \leqslant \mu \leqslant k-1$. Although we can pick any source cell, we will again choose $D_{*}=G_{00}$ for the sake of illustration. From the conformality condition (3.3) and using Lemma 3.3 , it is not difficult to see that the representation formula we will obtain is independent of the flow system. Hence, we will choose a main stream flowing to the right crossing $x-x_{1}=0, \ldots, x-x_{m}=0$ into the cells $G_{10}, \ldots, G_{m 0}$ consecutively, and a main stream flowing along the positive $y$-axis crossing $y-y_{1}=0, \ldots, y-y_{n}$ $=0$ into the cells $G_{01}, \ldots, G_{0 n}$ consecutively. The branches of this flow system will be travelling from each of the cells $G_{10}, \ldots, G_{m 0}$ parallel to the north-bound main stream into the remaining cells of the partition $\Delta_{m n}$. Hence, to reach $G_{i j}$ from $D_{*}=G_{00}$, the stream first travels eastward crossing $x-x_{1}=0, \ldots, x-x_{i}=0$ and then northward crossing $y-y_{1}=0, \ldots, y-y_{j}=0$. Suppose that the restriction of $s$ on $D_{*}$ is $p_{k}^{*} \in \mathbf{P}_{k}$. Then for $(x, y) \in G_{i 0}$, we have

$$
s(x, y)=p_{k}^{*}(x, y)+\sum_{p=1}^{i}\left(x-x_{p}\right)^{\mu+1} b_{p}(x, y)
$$


by using Lemma 2.2. Similarly, for $(x, y) \in G_{0 j}$, we have

$$
s(x, y)=p_{k}^{*}(x, y)+\sum_{l=1}^{j}\left(y-y_{l}\right)^{\mu+1} c_{l}(x, y),
$$

where $b_{1}, \ldots, b_{l}, c_{1}, \ldots, c_{1} \in \mathbf{P}_{k-\mu-1}$ are smoothing cofactors. Call $c_{l}=c_{0 l}$ and denote the smoothing cofactors from $G_{l, l-1}$ to $G_{i, l}$ by $c_{i l}$. Then by using Lemma $3.3 i$ times, we have

$$
c_{l l}(x, y)=c_{l}(x, y)+\sum_{p=1}^{l}\left(x-x_{p}\right)^{\mu+1} d_{p l}(x, y) \text {. }
$$

Hence, if $(x, y) \in G_{i j}$, we have

$$
\begin{aligned}
s(x, y)= & p_{k}^{*}(x, y)+\sum_{p=1}^{i}\left(x-x_{p}\right)^{\mu+1} b_{p}(x, y) \\
& +\sum_{l=1}^{j}\left(y-y_{l}\right)^{\mu+1}\left[c_{l}(x, y)+\sum_{p=1}^{i}\left(x-x_{p}\right)^{\mu+1} d_{p l}(x, y)\right] .
\end{aligned}
$$

By using the plus notation, we have

$$
\begin{aligned}
s(x, y)= & p_{k}^{*}(x, y)+\sum_{p=1}^{m} b_{p}(x, y)\left(x-x_{p}\right)_{+}^{\mu+1}+\sum_{l=1}^{n} c_{l}(x, y)\left(y-y_{l}\right)_{+}^{\mu+1} \\
& +\sum_{p=1}^{m} \sum_{l=1}^{n} d_{p l}(x, y)\left(x-x_{p}\right)_{+}^{\mu+1}\left(y-y_{l}\right)_{+}^{\mu+1} .
\end{aligned}
$$

Here, we have of course used the conformality condition at every grid-point. That is, we have the following result.

THEOREM 3.1. Any bivariate spline function $s$ in $S_{k}^{\mu}\left(\Delta_{m n}\right), 0 \leqslant \mu \leqslant k-1$, has the following unique representation:

$$
\begin{aligned}
s(x, y)= & p_{k}^{*}(x, y)+\sum_{p=1}^{m} b_{p}(x, y)\left(x-x_{p}\right)_{+}^{\mu+1}+\sum_{l=1}^{n} c_{l}(x, y)\left(y-y_{l}\right)_{+}^{\mu+1} \\
& +\sum_{p=1}^{m} \sum_{l=1}^{n} d_{p l}(x, y)\left(x-x_{p}\right)_{+}^{\mu+1}\left(y-y_{l}\right)_{+}^{\mu+1}
\end{aligned}
$$

where $p_{k}^{*} \in \mathbf{P}_{k}, b_{p}$ and $c_{l} \in \mathbf{P}_{k-\mu-1}$ and $d_{p l} \in \mathbf{P}_{k-2 \mu-2}$. If, in particular, $\mu>$ $(k-2) / 2$, then

$$
s(x, y)=p_{k}^{*}(x, y)+\sum_{p=1}^{m} b_{p}(x, y)\left(x-x_{p}\right)_{+}^{\mu+1}+\sum_{l=1}^{n} c_{l}(x, y)\left(y-y_{l}\right)_{+}^{\mu+1}
$$

Here and throughout this paper we use the notation $\mathbf{P}_{l}=\{0\}$ when $l<0$. There are many important consequences of this theorem. We first note that since the tensor-product spline functions of order $((\mu+2),(\mu+2))$ are dense in $X$, where 
$X=L_{p}(D)$ if $1 \leqslant p<\infty$ and $X=C(\bar{D})$ if $p=\infty$ as we defined earlier, we have the following

THEOREM 3.2. The closure in $X$ of the union of $S_{k}^{\mu}(\Delta)$ over all rectangular grid partitions $\Delta$ of $D$ is all of $X$ if and only if $\mu \leqslant(k-2) / 2$. If $\mu>(k-2) / 2$, then the closure is the space of all functions

$$
p_{k}(x, y)+q_{k-\mu-1}(x, y) f(x)+r_{k-\mu-1}(x, y) g(y),
$$

where $p_{k} \in \mathbf{P}_{k}$ and $q_{k-\mu-1}, r_{k-\mu-1} \in \mathbf{P}_{k-\mu-1}$.

We next study the nature of locally supported bivariate spline functions in terms of the normalized $B$-splines.

THEOREM 3.3. The bivariate spline space $S_{k}^{\mu}\left(\Delta_{m n}\right), 0 \leqslant \mu \leqslant k-1$, has dimension $\frac{1}{2}\left[(k+1)(k+2)+(m+n)(k-\mu)(k-\mu+1)+m n(k-2 \mu-1)_{+}(k-2 \mu)\right]$. For $\mu>(k-2) / 2$, it is spanned by

$$
\mathbb{Q}_{1}=\left\{x^{u} y^{v}, x^{a} y^{b} B_{i, \mu+2}(x), x^{c} y^{d} C_{j, \mu+2}(y)\right\},
$$

where $k-\mu-1 \leqslant u, v \leqslant \mu$ and $2 k-2 \mu-1 \leqslant u+v \leqslant k, i=1, \ldots, m+\mu+2$, $j=1, \ldots, n+\mu+2, \quad 0 \leqslant a+b \leqslant k-\mu-1,0 \leqslant c+d \leqslant k-\mu-1$. For $\mu \leqslant$ $(k-2) / 2$, it is spanned by

$$
\mathbb{H}_{2}^{\prime}=\left\{x^{k-2 \mu-1+a} y^{b} B_{i, \mu+2}(x), y^{k-2 \mu-1+c} x^{d} C_{j, \mu+2}(y), x^{u} y^{v} B_{p, \mu+2}(x) C_{q, \mu+2}(y)\right\},
$$

where $0 \leqslant a+b \leqslant \mu, 0 \leqslant c+d \leqslant \mu ; i, p=1, \ldots, m+\mu+2 ; j, q=1, \ldots, n+\mu+$ 2 , and $0 \leqslant u+v \leqslant k-2 \mu-2$.

The dimension of $S_{k}^{\mu}\left(\Delta_{m n}\right)$ can be found easily using the representation formula (3.4), where the spanning functions are clearly linearly independent. For computational and theoretical purposes it is advantageous to keep all the normalized $B$-splines $B_{i, \mu+2}(x)$ and $C_{j, \mu+2}(y)$. However, in doing so, we must also retain all coefficients $x^{a} y^{b}, 0 \leqslant a+b \leqslant k-\mu-1$ of $B_{i, \mu+2}(x)$ and $C_{j, \mu+2}(y)$ in the case $\mu>(k-2) / 2$, in order to generate all the spline elements $x^{a} y^{b}\left(x-x_{i}\right)_{+}^{\mu+1}$ and $x^{a} y^{b}\left(y-y_{j}\right)_{+}^{\mu+1}, 0 \leqslant a+b \leqslant k-\mu-1, i=1, \ldots, m+\mu+2$, and $j=1, \ldots, n+$ $\mu+2$. The elements $x^{a} y^{b} B_{i, \mu+2}(x)$ and $x^{c} y^{d} C_{j, \mu+2}(y), 0 \leqslant a+b \leqslant k-\mu-1$ and $0 \leqslant c+d \leqslant k-\mu-1$, have already generated the polynomials $x^{a+u} y^{b}$ and $x^{c} y^{d+v}$, $0 \leqslant u, v \leqslant \mu+1,0 \leqslant a+b \leqslant k-\mu-1$ and $0 \leqslant c+d \leqslant k-\mu-1$. For $\mu \leqslant$ $(k-\mu) / 2$, in order to generate all bivariate splines $x^{a} y^{b}\left(x-x_{i}\right)_{+}^{\mu+1}\left(y-y_{j}\right)_{+}^{\mu+1}$, $0 \leqslant a+b \leqslant k-2 \mu-2, i=1, \ldots, m, j=1, \ldots, n$, we must again keep all the coefficients $x^{a} y^{b}, 0 \leqslant a+b \leqslant k-2 \mu-2$, of $B_{i, \mu+2}(x) C_{j, \mu+2}(y)$. In doing so, we have already generated all the splines $x^{u} y^{v}\left(x-x_{i}\right)_{+}^{\mu+1}$ and $x^{p} y^{q}\left(y-y_{j}\right)_{+}^{\mu+1}$, with the exception of $x^{k-2 \mu-1+a} y^{b}\left(x-x_{i}\right)_{+}^{\mu+1}$ and $y^{k-2 \mu-1+a} x^{b}\left(y-y_{j}\right)_{+}^{\mu+1}$, where $0 \leqslant$ $a+b \leqslant \mu$ and $i=1, \ldots, m+\mu+2, j=1, \ldots, n+\mu+2$. All the polynomials in $\mathbf{P}_{k}$ have already been generated. That is, we have shown that $Q_{1}$ and $Q_{2}$ are the "smallest" spanning sets of $S_{k}^{\mu}\left(\Delta_{m, n}\right)$ for $u>(k-2) / 2$ and $\mu \leqslant(k-2) / 2$, respectively, if we want to use all of the $B$-splines $B_{i, \mu+2}(x), C_{j, \mu+2}(y)$, and $B_{i, \mu+2}(x) C_{j, \mu+2}(y)$. It is obvious that the spanning sets $Q_{1}$ and $\mathbb{Q}_{2}$ are much larger than the dimension of $S_{k}\left(\mu_{m n}\right)$. But with the normalized $B$-splines, it is more convenient to construct efficient approximants. 
4. Extensions and Remarks. Rectangular grid partitions are useful when the domain is rectangular. However, in applications such as studying stress, strength and pressure in constructions of dams and other structural designs where the domains of interest are polygonal, it is necessary to use cross-cut partitions with more than two directions in order to obtain good results near the boundary. A line segment is called a cross-cut of $D$ if it divides $D$ into two cells and its endpoints lie on $\partial D$, the boundary of $D$. A grid $\Delta$ of a finite number of cross-cuts of $D$ is called a cross-cut grid partition of $D$. Hence, each grid-segment (or edge) of a cell is a segment of a cross-cut of $\Delta$. Note that if $D$ is not convex, more than one cross-cut may lie on the same line. A cross-cut grid partition is said to be a simple cross-cut grid partition if no more than two cross-cuts meet at a grid-point.

We summarize some of the main results which are generalizations of those in the above section to an arbitrary simple cross-cut grid partition. Let

$$
\eta(t)= \begin{cases}\frac{1}{2}(t+1)(t+2) & \text { if } t \geqslant 0 \\ 0 & \text { if } t<0\end{cases}
$$

First we give the dimension of the smooth bivariate spline space.

LEMMA 4.1. Let $\Delta$ be a simple cross-cut grid partition of a simply connected domain $D$, consisting of $L$ cross-cuts and $V$ grid-points. Then the dimension of $S_{k}^{\mu}(\Delta, D)$ is

$$
\eta(k)+L \eta(k-\mu-1)+V \eta(k-2 \mu-2) .
$$

To give an explicit basis for $S_{k}^{\mu}(\Delta, D)$, it is more convenient to group the cross-cuts of the partition. Let $\left(a_{1}, b_{1}\right), \ldots,\left(a_{N}, b_{N}\right)$ be pairwise linearly independent ordered pairs, $\mathbf{c}=\left[c_{i \rho}\right]$ a matrix of numbers,

$$
\Gamma_{i \rho}: a_{i} x+b_{i} y+c_{i \rho}=0
$$

a collection of straight lines, and let the simple cross-cut grid partition $\Delta=\Delta(N, \mathbf{c})$ of $D$ consist of cross-cuts

$$
l_{i j}=l_{i \rho j}, \quad j=1, \ldots, t_{i} \text { and } i=1, \ldots, N,
$$

where each $l_{i \rho j}$ is a segment of the line $\Gamma_{i \rho}$. Each cross-cut $l_{i j}=l_{i \rho j}$ divides $D$ into two cells: the one not containing the source cell $D_{*}$ will be denoted by $D_{i j}=D_{i \rho j}$ and the other by $D_{i j}^{\prime}=D_{i \rho j}^{\prime}$. We define the function $\left(l_{i j}\right)_{\#}=\left(l_{i \rho j}\right)_{\#}$ by

$$
\left(l_{i j}\right)_{\#}(x, y)= \begin{cases}a_{i} x+b_{i} y+c_{i \rho} & \text { if }(x, y) \in D_{i j}, \\ 0 & \text { if }(x, y) \in D_{i j}^{\prime} \cup l_{i j},\end{cases}
$$

and $\left(l_{i j}\right)_{\#}^{\mu+1}(x, y)=\left[\left(l_{i j}\right)_{\#}(x, y)\right]^{\mu+1}$. In addition, define the index sets

$$
\Omega(i, r)=\left\{(j, s): l_{i j} \text { and } l_{r s} \text { have a common grid-point lying in } D\right\},
$$

and let $\omega(i, r)$ be the cardinality of $\Omega(i, r)$. We have the following

THEOREM 4.1. Let $N \geqslant 2$ and $0 \leqslant \mu<k$. Then the collection

$$
e=\left\{x^{a} y^{b}, x^{c} y^{d}\left(l_{i j}\right)_{\#}^{\mu+1}, x^{u} y^{v}\left(l_{h j}\right)_{\#}^{\mu+1}\left(l_{r s}\right)_{\#}^{\mu+1}\right\},
$$

where $0 \leqslant a+b \leqslant k, 0 \leqslant c+d \leqslant k-\mu-1,0 \leqslant u+v \leqslant k-2 \mu-2, h \neq r$, and $(j, s) \in \Omega(h, r)$, is a basis of $S_{k}^{\mu}(\Delta)$. 
Here the functions $x^{u} y^{v}\left(l_{h j}\right)_{\#}^{\mu+1}\left(l_{r s}\right)_{\#}^{\mu+1}$ are to be deleted from $\bigodot$ if $k<2 \mu+2$. If $N=2, D$ is the unit square $\{(x, y): 0<x, y<1\}, \Gamma_{1, \rho}: x+c_{1 \rho}=0$ and $\Gamma_{2, \rho}$ : $y+c_{2 \rho}=0\left(x_{\rho}=-c_{1 \rho}\right.$ and $\left.y_{\rho}=-c_{2 \rho}\right)$, and the source cell $D_{*}$ is $\{(x, y): 0<x<$ $\left.x_{1}, 0<y<y_{1}\right\}$, then the "\#" functions become the " + " functions and Theorem 3.1 is a particular case of Theorem 4.1.

As an immediate consequence, we can decide how "well" the bivariate splines in

$$
\hat{S}_{k}^{\mu}(N)=\bigcup_{\mathbf{c}} S_{k}(\Delta(N, \mathbf{c}))
$$

approximate. That is, we have the following

COROLlaRY 4.1. The closure of $\hat{S}_{k}^{\mu}(N)$ in the topology of uniform convergence on compact subsets of $D$ is $C(D)$ if and only if $\mu \leqslant(k-2) / 2$. If $\mu>(k-2) / 2$, the closure of $\hat{S}_{k}^{\mu}(N)$ is the space of continuous functions of the form

$$
p_{k}(x, y)+q_{1}(x, y) f_{1}\left(a_{1} x+b_{1} y\right)+\cdots+q_{N}(x, y) f_{N}\left(a_{N} x+b_{N} y\right),
$$

where $f_{1}, \ldots, f_{N}$ are continuous functions of one variable, $q_{1}, \ldots, q_{N} \in \mathbf{P}_{k-\mu-1}$ and $p_{k} \in \mathbf{P}_{k}$.

Of course, if $D$ is bounded, the supremum normed or $L_{p}$ normed topologies can be used.

An important problem is to find a nonnegative locally supported basis (or even

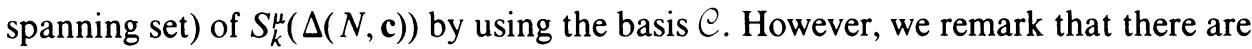
no locally supported bivariate spline functions in $C^{\mu}(D)$ for "large" $\mu$ as in the following:

Proposition 4.1. There are no locally supported functions in $S_{k}(\Delta(N, \mathbf{c}))$ for $\mu>(k-2) / 2$.

In this paper we have not considered the important case where the cross-cut grid partitions are not simple and the even more important case where $\Delta$ is an arbitrary grid partition. We believe, however, that our techniques can be extended to study these important problems as well as the structure of bivariate spline functions with grid partitions consisting of arbitrary irreducible algebraic curves. If the cross-cut grid partition $\Delta$ is not simple, it is in general necessary to invert very complicated matrices to determine the basis functions of $S_{k}^{\mu}(\Delta, D)$ explicitly. These problems will be studied at a later date. We are grateful to the referee for many valuable suggestions on the revision of the manuscript and for supplying us with some references. We would also like to thank Professor C. de Boor for several useful comments and for sending us a copy of [7]. This very interesting paper [7] answers the important question "How effective are multivariate splines in approximation?" instead of studying the dimension and basis problem as we do in the present paper.

\footnotetext{
Department of Mathematics Texas A \& M University College Station, Texas 77843

Institute of Mathematics and Department of Mathematics Jilin University Changchun, Jilin, China
} 
1. R. E. Barnhill, G. Birkhoff \& W. J. Gordon, "Smooth interpolation in triangles," J. Approx. Theory, v. 8, 1973, pp. 114-128.

2. R. K. Beatson, “Convex approximation by splines,” SIAM J. Math. Anal. (To appear.)

3. G. Birkhoff \& H. Garabedian, "Smooth surface interpolation," J. Math. Phys., v. 39, 1960, pp. $258-268$.

4. C. DE Boor, “On calculating with $B$-splines,” J. Approx. Theory, v. 6, 1972, pp. 50-62.

5. C. DE Boor, "Splines as linear combination of $B$-splines," in Approximation Theory II (G. (G. Lorentz, C. K. Chui, L. L. Schumaker, Eds.), Academic Press, New York, 1976, pp. 1-47.

6. C. DE Boor, A Practical Guide to Splines. Springer-Verlag, New York, 1978.

7. C. DE BOOR \& R. DeVORE, “Approximation by smooth multivariate splines.” (In manuscript.)

8. C. K. Chui, P. W. Smith \& J. D. WARD, "Degree of $L_{\text {. }}$ approximation by monotone splines," SIAM J. Math. Anal., v. 11, 1980, 436-447.

9. C. K. Chui, P. W. Smith \& J. D. Ward, "Monotone approximation by spline functions," in Quantitative Approximation (R. A. DeVore and K. Scherer, Eds.), Academic Press, New York, 1980.

10. C. K. Chui \& R. H. WANG, "Bases of bivariate spline spaces with cross-cut grid partitions," $J$. Math. Res. Exposition, v. 2, 1982, pp. 1-3.

11. S. A. Coons, "Surface patches and B-spline curves," in Computer Aided Geometric Design (R. E. Barnhill and R. F. Riesenfeld, Eds.), Academic Press, New York, 1974.

12. W. Dahmen, “On multivariate $B$-splines,” SIAM J. Numer. Anal., v. 17, 1980, pp. 179-190.

13. W. Dahmen, R. DeVore \& K. SCherer, “Multidimensional spline approximations,”, SIAM J. Numer. Anal., v. 17, 1980, pp. 380-402.

14. W. Dahmen \& C. A. Micchelli, On Limits of Multivariate B-Splines, MRC Technical Summary Report \#2114, University of Wisconsin, 1980.

15. R. A. DeVore, "Monotone approximation by splines," SIAM J. Math. Anal., v. 8, 1977. pp. $891-905$.

16. T. DUPONT \& R. ScotT, "Polynomial approximation of functions in Sobolev spaces," Math. Comp., v. 34, 1980, pp. 441-463.

17. W. J. GORDON, "Blending-function methods of bivariate and multivariate interpolation and approximation," SIAM J. Numer. Anal., v. 8, 1971, pp. 158-177.

18. C. A. Hall, "Bicubic interpolation over triangles," J. Math. Mech., v. 19, 1969, pp. 1-11.

19. C. A. MicChelli, "A constructive approach to Kergin interpolation in $R^{h}$ : Multivariate $B$-splines and Lagrange interpolation," Rocky Mountain J. Math. (To appcar.)

20. C. A. Micchelli, "On numerically efficient methods for computing multivariate $B$-splines," in Multivariate Approximation Theory (W. Schempp and K. Zeller, Eds.), ISNM 51, Birkhauser-Verlag. Basel, 1979.

21. M. Munteanu \& L. L. Schumaker, "Direct and inverse theorems for multi-dimensional spline approximation," Indiana Univ. Math. J., v. 23, 1973, pp. 46I-470.

22. I. J. SCHOENBERG, "Contributions to the problem of approximation of equidistant data by analytic functions," Quart. Appl. Math., v. 4, 1946, pp. 45-99, 112-141.

23. M. H. Schultz, Spline Analysis, Prentice-Hall, Englewood Cliffs, N.J., 1973.

24. L. L. Schumaker, "Fitting surfaces to scattered data," in Approximation Theory. II (G. G. Lorentz. C. K. Chui and L. L. Schumaker, Eds.), Academic Press, New York, 1976.

25. L. L. Schumaker, "On the dimension of spaces of piecewise polynomials in two variables," in Multivariate Approximation Theory (W. Schempp and K. Zeller, Eds.), ISBN 51, Birkhauser-Verlag, Basel, 1979.

26. G. Strang, "The dimension of piecewise polynomials and one-sided approximation," in Proc. Conf. Numerical Solution of Differential Equations (Dundee 1973), Lecture Notes in Math., \#365. Springer-Verlag, Berlin and New York, 1974, pp. 144-152.

27. G. Strang, "Piecewise polynomials and finite element methods," Bull. Amer. Math. Soc., v. 79. 1973, pp. 1128-1137.

28. R. J. Walker, Algebraic Curves, Princeton Univ. Press, Princeton, N.J., 1950.

29. R. H. WANG, "The structural characterization and interpolation for multivariate splines," $A$ cta Math. Sinica, v. 18, 1975, pp. 91-106.

30. R. H. WANG, "On the analysis of multivariate splines in the case of arbitrary partition," Sci. Sinica (Math. I), 1979, pp. 215-226.

31. R. H. WANG, "On the analysis of multivariate splines in the case of arbitrary partition. II," Num. Math. J. Chinese Univ., v. 2, 1980, pp. 78-81.

32. R. H. Wang, S. Z. Liang \& Y. S. Chou, Approximation of Functions of Several Variables (in Chinese), Science Press, Peking. (To appear.) 